\title{
Electromyographic analysis of shoulder girdle muscles in volleyball throw: A reliability study
}

\author{
Shahrzad Zandi ${ }^{1}$, Reza Rajabi $^{1 *}$, Mohammadali Mohseni-Bandpei ${ }^{2,3}$, Hooman Minoonejad $^{1}$ \\ ${ }^{1}$ Department of Health and Sports Medicine, Faculty of Physical Education and Sport Sciences, University of Tehran, \\ Tehran, Iran; ${ }^{2}$ Iranian Research Centre on Aging, Department of Physiotherapy, University of Social Welfare and \\ Rehabilitation Sciences, Tehran, Iran; ${ }^{3}$ University Institute of Physical Therapy, Faculty of Allied Health Sciences, \\ University of Lahore, Lahore, Pakistan
}

\section{Summary}

Study aim: The aim of this study was to investigate the reliability of the electromyographic activity of selected shoulder girdle muscles during the overhead volleyball throw.

Material and methods: The test-retest reliability of EMG activity of selected shoulder muscles during an overhead volleyball throw was investigated in 15 non-symptomatic university-level female volleyball players for within-day sessions (with a onehour interval) and between-day sessions (with a one-week interval). Time broadness (a measure of coordination) and root mean square of electromyography signals were obtained.

Results: A high within-day (0.85-0.99) and moderate to high between-day (0.68-0.93) intraclass correlation coefficient for normalized RMS activity and a high within-day and between-day intraclass correlation coefficient ( 0.94 and 0.80 ; respectively) for time broadness were observed. Absolute agreement of measurements had small values (0.15-1.96). Trends toward higher intraclass correlation coefficient values and lower standard error of measurements, minimum detectable change, mean differences and limits of agreements values were observed for within-day reliability in all test results compared with between-day reliability.

Conclusions: The results suggest that the activity of shoulder muscles can be reliably assessed during the overhead volleyball throw with the described procedure both in the amplitude domain (normalized average root mean square) and the time domain (time broadness of the activities).

\section{Keywords: Reliability - Electromyography - Volleyball throw - Shoulder girdle muscles}

\section{Introduction}

Electromyography (EMG) is a commonly used and valid tool for study of the characteristics of muscle activities [8]. It has been used as a quantitative instrument to study muscle activity patterns in shoulder rehabilitation [14] as well as to analyze shoulder muscle activity $[13,14,17]$, activation onset time $[18,27]$ and coordination $[9,14,15]$ during sport activities in both healthy and injured shoulders $[11,17]$.

After several years of heavy training, the risk of shoulder injuries may increase in overhead thrower athletes [6]. Shoulder instability is one of the most common injuries and can lead to other secondary pathologies over time $[4,6,19]$, and thus, put the health and career of the athlete in danger.
Increasing the activity and coordination of muscles responsible for the functional stability of the shoulder joint may lead to injury prevention by maintaining the humeral head in the glenoid fossa $[20,28]$. In case of instability, rotator cuff muscles, which are the main parts of core muscles of the shoulder girdle, have the most significant role in stabilizing the shoulder joint $[2,11,22]$. Therefore, it is important to observe and trace their function in high risk activities such as an overhead volleyball serve or spike.

Although many studies have been conducted to examine muscle activity in a thrower's shoulders using dynamic EMG [2, 10, 16, 22, 23], few of them have focused on shoulder muscle activity during functional tasks which are more similar to the sport activity $[14,15,17,18,26]$. Illyés and Kiss (2009) have reported the activity and coordination of shoulder muscles in professional javelin throwers during 
overhead throw of a tennis ball $[2,10,14,22]$. Although they used and suggested a newly introduced demonstrating parameter of muscle coordination, they did not report the reliability of EMG recordings during the task. Kelly and colleagues (2002) have also studied shoulder muscles EMG during the functional football throw in professional players. They reported little variation in EMG activation between subjects during the phases of throw motion, but still, they indicated no information about the reliability of EMG. Amongst the few studies that have reported the reliability of EMG of shoulder muscles, a lack of functional tasks is also represented [1, 21, 29]. Andersen and colleagues (2014) have investigated between-day reliability of root mean square (RMS) of EMG signals in activity of upper trapezius and anterior deltoid muscles in static conditions (isometric submaximal contractions during flexion $45^{\circ} / 90^{\circ}$, abduction $45^{\circ} / 115^{\circ}$, and internal/external rotation) and obtained acceptable reliability (ICC $=0.82-0.92)$ and proper limits of agreement with near to zero mean difference of the measurements (mean difference $=0.25-0.70$ ), but these results are limited only to these isometric tasks. Ludewig and Cook (2000) reported moderate to high $(\mathrm{ICC}=0.73-0.90)$ reliability of measuring RMS activity of upper trapezius, lower trapezius and serratus anterior muscles in scaption movement after 2 days. In a similar study, Seitz and Uhl (2012) investigated the within-day and between-day reliability of RMS activity of anterior deltoid, upper trapezius, lower trapezius and serratus anterior muscles in scaption movement. They obtained higher within-day reliability $(\mathrm{ICC}=0.96-0.99 ; \mathrm{SEM}=1.0-2.0$; $\mathrm{MDC}=1.3-2.9)$ compared to between-day reliability ( $\mathrm{ICC}=0.59-0.86 ; \mathrm{SEM}=2.3-8.3 ; \mathrm{MDC}=3.2-11.7)$. In both studies the movement was performed with the speed of one repetition every 4 seconds, which is far slower than the rapid arm movement in volleyball spikes or serves.

Although there are differences between laboratory conditions and match conditions, the overhead test is suggested as a valid functional test in volleyball, which has higher similarity to the athletic performance than the isolated-joint conditions [24], and despite some previous reports of the amount of muscle activity during the spike and overhead serve [26], there is a lack of evidence regarding the reliability of EMG recordings during these tasks so far. To the knowledge of the authors of the present study, no published evidence has been found regarding the EMG assessment of the shoulder during the volleyball throw. Development of a reliable functional task for recording EMG characteristics of shoulder girdle muscles would benefit many aspects of athletic training, rehabilitation and examination of the players. The purpose of this study was, therefore, to investigate the reliability of the EMG activity and coordination of selected shoulder girdle muscles during the overhead volleyball throw.

\section{Materials and methods}

\section{Subjects}

A relatively homogeneous study group, consisting of 15 female healthy university level volleyball players (wing spikers), was recruited for this study. Exclusion criteria included any history of shoulder pain, current upperextremity injury, and recent (6 months) back and/or neck injury. All participants signed a written informed consent form before participating in the study. The study protocol on human subjects was approved by the ethical review board of the University of Tehran. Sample size estimation indicated that with intra-rater intraclass correlation coefficients (ICCs) that exceeded 0.7, an alpha level of 5\%, a power of $80 \%$, and an effect size of $0.5,15$ subjects were required [7]. Demographic characteristics of subjects are shown in Table 1.

\section{Electromyography}

The Megawin (ME6000-T16 Mega Electronics, Kuopio, Finland) System Biomonitor (10 times pre-amplified cables; gain = 1000) and Software "Megawin" version 3.1-b10 were used to record and conduct a band-pass filter $(20-500 \mathrm{~Hz})$ of the EMG signal with a $1000 \mathrm{~Hz}$ sampling rate. The skin was prepared by shaving then lightly abraded with fine sandpaper and cleaned with $70 \%$ isopropyl alcohol to minimize impedance. Self-adhesive $\mathrm{Ag} / \mathrm{AgCl}$ bipolar, dual surface electrodes (AQUA-TAC SKINTACT ECG Electrodes) were placed over the preparation sites in line with the muscle fibers. The electrodes were $0.0032 \times 0.0041 \mathrm{~m}$ with an inter-electrode distance of $0.03 \mathrm{~m}$. The same investigator attached all electrodes. Electrode placements and the absence of noticeable cross-

Table 1. Demographic characteristics of subjects

\begin{tabular}{lccc}
\hline & Mean \pm SD & Min & Max \\
\hline Age [years] & $20.93 \pm 1.94$ & 19.00 & 25.00 \\
Body mass [kg] & $58.27 \pm 5.96$ & 48.00 & 75.00 \\
Height [m] & $1.68 \pm 0.01$ & 1.66 & 1.72 \\
History of playing volleyball [years] & $6.80 \pm 2.93$ & 4.00 & 15.00 \\
\hline
\end{tabular}


talk were confirmed by evaluating the activity of each muscle with manual muscle tests [12]. Once EMG electrode placements had been confirmed, the sensors and leads were secured with adhesive tape to minimize movement artifacts. A biaxial electrogoniometer was also attached to the posterior surface of the shoulder (in $90^{\circ}$ abduction and $0^{\circ}$ rotation of the shoulder) in order to detect the initial acceleration phase of the throw [26].

The EMG data were collected from ten muscles - supraspinatus (SS), infraspinatus (IS), upper trapezius (UT), lower trapezius (LT), pectoralis major (PM), serratus anterior (SA), anterior deltoid (AD), posterior deltoid (PD), biceps brachii $(\mathrm{BB})$ and triceps brachii $(\mathrm{TB})$ - of each subject's dominant shoulder. The dominant shoulder was defined as the shoulder which is always used for spike in volleyball and was used for EMG data collection for each person.

The EMG electrodes were placed over the measured midpoint of the muscle belly using CRAM's instructions for electrode placement [8]. The electrode placements were as follows (Fig. 1): SS was located directly above the spine of the scapula, over the suprascapular fossa. IS was located parallel to and approximately $4 \mathrm{~cm}$ below the spine of the scapula, on the lateral aspect, over the infrascapular fossa. UT was located along the ridge of the shoulder,

a.

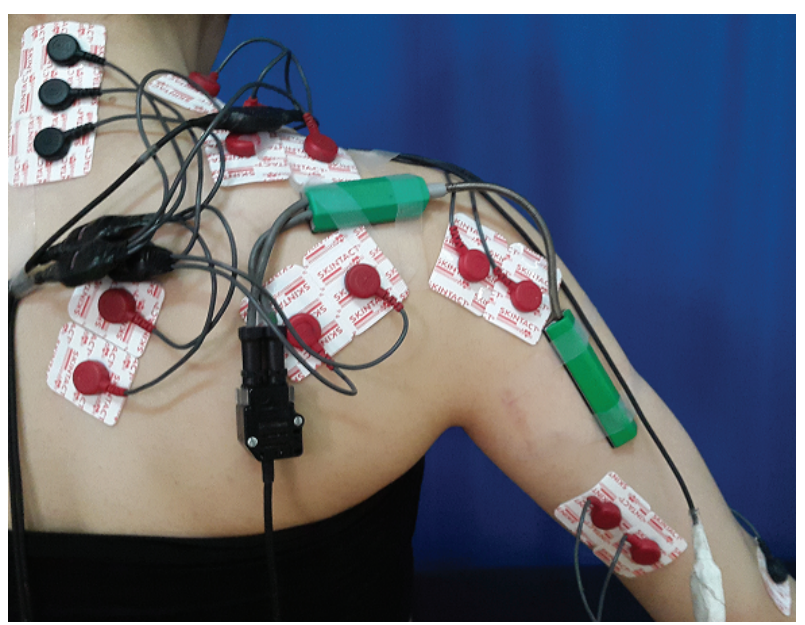

b.

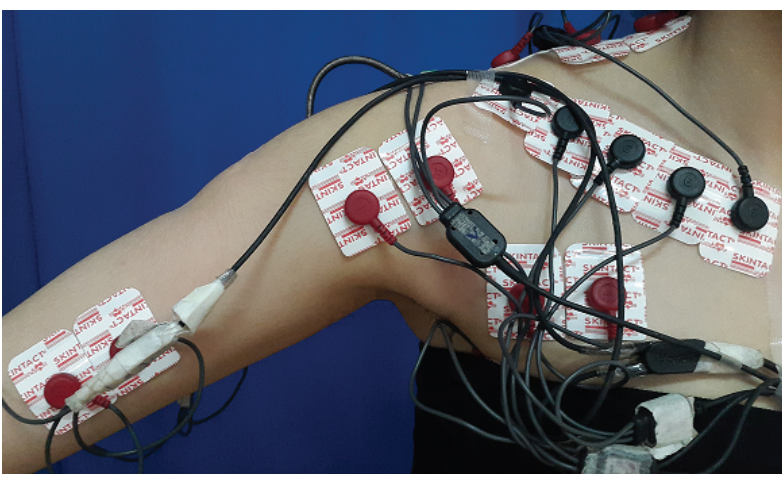

Fig. 1. Electrode placements (a. posterior view; b. anterior view) slightly lateral to and one-half the distance between the cervical spine at C-7 and the acromion. LT was located in the interscapular region at an oblique angle, approximately $5 \mathrm{~cm}$ down from the scapular spine next to the medial edge of the scapula at a 55-degree oblique angle. PM was located horizontally on the chest wall and $2 \mathrm{~cm}$ medial to the anterior axillary fold (for the sternal portion of the muscle). SA was located horizontally just below the axillary area, at the level of the inferior tip of the scapula, and just medial to the latissimus dorsi border. AD was located on the anterior aspect of the arm, approximately $4 \mathrm{~cm}$ below the clavicle, parallel to the muscle fibers. PD was also located approximately $2 \mathrm{~cm}$ below the lateral border of the spine of the scapula and angled at an oblique angle toward the arm. BB was located at the center of the muscle mass in the middle anterior aspect of the upper arm. TB was also located $2 \mathrm{~cm}$ medial from the midline of the arm, approximately $50 \%$ of the distance between the acromion and the olecranon.

\section{Procedure}

Subjects performed a rapid standing volleyball throw of a volleyball which was hung from the ceiling. The height of the ball was adjusted according to the height of the subject's dominant hand palm in a standing position with $180^{\circ}$ shoulder flexion and full elbow extension. Subjects stood with their dominant shoulder just under the ball. The place of the feet was marked on the ground. Subjects were asked to hit the ball in a forward direction as fast as they could, in the position that was natural for them with the technique they wished [14]. Each measurement consisted of five trials which were paced using a metronome. They were instructed not to change the position of their feet or their head during the measurement and to relax their muscles during the gap between the trials. The investigator monitored the EMG data acquisition online for detecting baseline noise or motion artifacts. Three out of these five trials were used for further analysis. After the electrode placement, the subjects performed a five-minute local and global warm-up. After warm-up and familiarization with the test, the first measurement was recorded. After approximately 1 hour, the second measurement was performed for calculating the within-day reliability. The electrodes remained on the skin only for the second measurement. A third measurement was also performed 1 week later for calculating the between-day reliability. In the second and third measurements both the investigator and the subject were blind to the results of previous measurements.

\section{Data reduction}

All signal processing was carried out using MATLAB software (The MathWorks Inc., Natick, Massachusetts, USA). The beginning and the end of the motion cycle were calculated for each trial. The beginning moment was 
considered the beginning of the acceleration phase, which was recorded using an electrogoniometer, and the end of the cycle was considered the maximum of the offsets of muscles $[14,15,17]$. The offset time of muscles was considered the moment that muscle activity amplitude stayed for more than $25 \mathrm{~ms}$ lower than the amount of mean baseline noise plus 3 times the standard deviation of the baseline activity $[2,18,27]$. The mean and standard deviation of the baseline activity were calculated during a window of $200 \mathrm{~ms}$ of relaxed position observed prior to the trial. The RMS values of EMG signals were calculated for consecutive segments of $25 \mathrm{~ms}$ during the motion cycle after conducting an order 10 low-pass digital filter with normalized cutoff frequency 0.1 . In order to allow comparisons of the activity in specific muscles and the activity in specific muscles among different measures and individuals, the EMG was normalized. RMS normalized values were calculated for each muscle as a percentage of the peak value observed in the cycle $[2,5,22]$.

Time broadness is the time elapsed (in percent) of the motion cycle between the peak of the first muscle to reach maximal activity and the peak of the last muscle to reach maximal activity. Time broadness can describe to what extent the muscles are involved in producing a motion simultaneously during a motion cycle. Time broadness provides indirect information on muscle coordination $[14,15]$. Using MATLAB software, the relative time of each muscle's peak value was calculated and the duration between first and last recorded peak value for all 10 muscles was normalized to the motion cycle (in percent) and considered as the time broadness.

\section{Statistical analysis}

The 1-sample K-S statistical test was used for data normality distribution testing. Intraclass correlation coefficients (ICC) were used to assess within-day (between the first and second measurements) and between-day (between the first and third measurements) reliability of measuring muscle activities (normalized RMS) and the time broadness among peak muscle activities. We considered the reliability coefficient values of 0.80 to 1.00 to indicate high, 0.60 to 0.80 to indicate moderate, and less than 0.59 to indicate questionable reliability, as was previously recommended [25]. The standard error of measurements (SEM) as an absolute index of reliability was also calculated using SD and ICC [30]. The SEM can be used to determine the minimum difference (MD) to be considered 'real' [30]. The formulas used for SEM and MD are shown in equation (1) and (2), respectively. Once the MD is calculated, then any change in a subject's score, either above or below the previous score, greater than the MD is considered real. More precisely, for all people whose differences on repeated testing are greater than or equal to the MD, 95\% of them would reflect real differences [30].

$$
\begin{gathered}
\mathrm{SEM}=\mathrm{SD} \sqrt{1-\mathrm{ICC}} \\
\mathrm{MD}=\mathrm{SEM} \times 1.96 \times \sqrt{2}
\end{gathered}
$$

As has been suggested by Bland and Altman (2010), a high correlation does not mean that the two sets of data agree, as correlation measures the strength of a relation between two sets of data, not the agreement between them [3]. Therefore, an additional method, as described by Bland and Altman (2010), was used to demonstrate how closely the measurements agree on different occasions. They recommended use of the $95 \%$ confidence interval $(95 \% \mathrm{CI})$ of the range of differences (limits of agreement) between the two measurements. In order to calculate the limits of agreement, the mean differences of each pair of measurements and the standard deviation were calculated. According to these researchers the mean difference of the measurements, as a measure of absolute agreement, should have a value near to zero and the limits of agreement lie two standard deviations below and above the mean difference, inside which $95 \%$ of the differences should be [3].

\section{Results}

The mean and standard deviation of the EMG normalized RMS average of the activity of 10 muscles and normalized time broadness of the muscle activities recorded from three measurements are reported in Table 2. The ICC values, SEM, MD and the mean difference of the measurements for within-day and between-day reliability are shown in Table 3.

According to criteria suggested by Richman et al. (1980), high within-day ( $\mathrm{ICC}=0.85-0.99)$ and moderate to high between-day ( $\mathrm{ICC}=0.68-0.93$ ) reliability were observed for normalized RMS activity of all muscles. High within-day and between-day reliability $(\mathrm{ICC}=0.94$ and 0.80 ; respectively) was also obtained for normalized time broadness of muscle activities. A trend towards higher ICC values and lower SEM values was observed for within-day reliability in all the test results compared with between-day reliability. The absolute agreement of measurements, which is represented by the mean difference of the measurements, had small values (0.15-1.96) and was generally smaller in within-day compared to between-day reliability.

Figures 2 and 3 are examples that display an agreement between the measurements for RMS and time broadness for both occasions. The limits of agreement were defined as the mean difference of the two measurements \pm 2 standard deviations. The mean difference should be zero or no significant difference between two means. For example, Figure 2 shows a comparison of RMS between the first and second measurements (within-day) and first and third measurements (between-day). The mean differences were 
Table 2. The mean and standard deviation of EMG parameters recorded from three measurements

\begin{tabular}{|c|c|c|c|c|c|c|}
\hline & \multicolumn{2}{|c|}{$1^{\text {st }}$ measurement } & \multicolumn{2}{|c|}{$2^{\text {nd }}$ measurement } & \multicolumn{2}{|c|}{$3^{\text {rd }}$ measurement } \\
\hline & Mean & $\mathrm{SD}$ & Mean & $\mathrm{SD}$ & Mean & SD \\
\hline \multicolumn{7}{|l|}{ Normalized RMS average } \\
\hline Supraspinatus & 24.07 & 5.11 & 23.51 & 4.90 & 23.72 & 4.45 \\
\hline Infraspinatus & 26.44 & 6.92 & 26.40 & 4.91 & 27.38 & 6.03 \\
\hline Upper trapezius & 23.76 & 5.86 & 23.52 & 5.24 & 25.64 & 5.71 \\
\hline Lower trapezius & 18.80 & 4.66 & 18.91 & 4.29 & 19.71 & 3.28 \\
\hline Prctoralis major & 32.83 & 7.90 & 34.02 & 8.41 & 32.30 & 6.06 \\
\hline Serratus anterior & 30.87 & 6.50 & 30.34 & 6.18 & 30.40 & 4.88 \\
\hline Anterior deltoid & 29.58 & 6.42 & 29.96 & 7.75 & 29.45 & 7.07 \\
\hline Posterior deltoid & 20.95 & 4.12 & 20.81 & 3.59 & 20.57 & 3.17 \\
\hline Biceps brachii & 23.46 & 4.61 & 23.64 & 4.74 & 25.10 & 4.12 \\
\hline Triceps brachii & 26.83 & 5.18 & 27.13 & 4.97 & 26.63 & 5.56 \\
\hline Normalized time broadness & 17.01 & 1.44 & 17.10 & 1.45 & 17.29 & 1.77 \\
\hline
\end{tabular}

Table 3. Within-day and between-days reliability analysis of the measurements

\begin{tabular}{|c|c|c|c|c|c|c|c|c|c|}
\hline & & Cronbach's $\alpha$ & $\mathrm{r}$ & $\mathrm{ICC}$ & Sig. & SEM & MD & $\begin{array}{c}\text { Mean } \\
\text { difference }\end{array}$ & $\begin{array}{c}\text { Limits of } \\
\text { agreement }\end{array}$ \\
\hline \multicolumn{10}{|c|}{ Normalized RMS average } \\
\hline \multirow{2}{*}{ Supraspinatus } & $\mathrm{WD}^{*}$ & 0.998 & 0.998 & 0.99 & 0.001 & 0.75 & 2.10 & 0.29 & $-0.05 \sim 0.64$ \\
\hline & $\mathrm{BD}^{*}$ & 0.964 & 0.939 & 0.93 & 0.001 & 1.99 & 5.53 & 0.73 & $-0.29 \sim 1.76$ \\
\hline \multirow{2}{*}{ Infraspinatus } & WD & 0.956 & 0.970 & 0.92 & 0.001 & 2.69 & 7.46 & 0.90 & $-0.71 \sim 2.51$ \\
\hline & $\mathrm{BD}$ & 0.927 & 0.873 & 0.86 & 0.001 & 3.84 & 10.65 & 1.41 & $-0.52 \sim 3.35$ \\
\hline \multirow{2}{*}{ Upper trapezius } & WD & 0.937 & 0.886 & 0.88 & 0.001 & 2.98 & 8.27 & 1.01 & $-0.74 \sim 2.76$ \\
\hline & $\mathrm{BD}$ & 0.905 & 0.827 & 0.79 & 0.001 & 4.24 & 11.77 & 1.67 & $-0.14 \sim 3.50$ \\
\hline \multirow{2}{*}{ Lower trapezius } & WD & 0.942 & 0.894 & 0.89 & 0.001 & 2.29 & 6.36 & 0.84 & $-0.32 \sim 2.01$ \\
\hline & $\mathrm{BD}$ & 0.837 & 0.765 & 0.71 & 0.001 & 3.45 & 9.58 & 1.36 & $-0.04 \sim 2.77$ \\
\hline \multirow{2}{*}{ Pectoralis major } & WD & 0.968 & 0.941 & 0.93 & 0.001 & 3.38 & 9.36 & 1.17 & $-0.77 \sim 3.11$ \\
\hline & $\mathrm{BD}$ & 0.877 & 0.808 & 0.79 & 0.001 & 5.15 & 14.27 & 1.96 & $-0.37 \sim 4.30$ \\
\hline \multirow{2}{*}{ Serratus anterior } & WD & 0.946 & 0.899 & 0.90 & 0.001 & 3.20 & 8.88 & 1.11 & $-0.69 \sim 2.92$ \\
\hline & $\mathrm{BD}$ & 0.815 & 0.717 & 0.70 & 0.002 & 5.03 & 13.94 & 1.89 & $-0.46 \sim 4.24$ \\
\hline \multirow{2}{*}{ Anterior deltoid } & WD & 0.915 & 0.858 & 0.85 & 0.001 & 4.38 & 12.15 & 1.24 & $-1.83 \sim 4.31$ \\
\hline & $\mathrm{BD}$ & 0.887 & 0.801 & 0.80 & 0.001 & 4.72 & 13.08 & 1.73 & $-0.61 \sim 4.09$ \\
\hline \multirow{2}{*}{ Posterior deltoid } & WD & 0.977 & 0.963 & 0.95 & 0.001 & 1.28 & 3.55 & 0.47 & $-0.15 \sim 1.11$ \\
\hline & $\mathrm{BD}$ & 0.801 & 0.691 & 0.68 & 0.002 & 3.32 & 9.22 & 1.21 & $-0.46 \sim 2.89$ \\
\hline \multirow{2}{*}{ Biceps brachii } & WD & 0.956 & 0.915 & 0.92 & 0.001 & 2.11 & 5.85 & 0.71 & $-0.53 \sim 1.96$ \\
\hline & $\mathrm{BD}$ & 0.901 & 0.824 & 0.77 & 0.002 & 3.38 & 9.36 & 1.35 & $-0.00 \sim 2.72$ \\
\hline \multirow{2}{*}{ Triceps brachii } & WD & 0.966 & 0.935 & 0.93 & 0.001 & 1.96 & 5.43 & 0.70 & $-0.46 \sim 1.87$ \\
\hline & $\mathrm{BD}$ & 0.855 & 0.749 & 0.76 & 0.001 & 4.05 & 11.22 & 1.64 & $-0.11 \sim 3.39$ \\
\hline \multicolumn{10}{|c|}{ Normalized time broadness } \\
\hline & WD & 0.968 & 0.938 & 0.94 & 0.001 & 0.56 & 1.57 & 0.15 & $-0.26 \sim 0.56$ \\
\hline & $\mathrm{BD}$ & 0.893 & 0.823 & 0.80 & 0.001 & 1.14 & 3.18 & 0.39 & $-0.25 \sim 1.05$ \\
\hline
\end{tabular}

* WD: Within-day. BD: Between-day 
a.

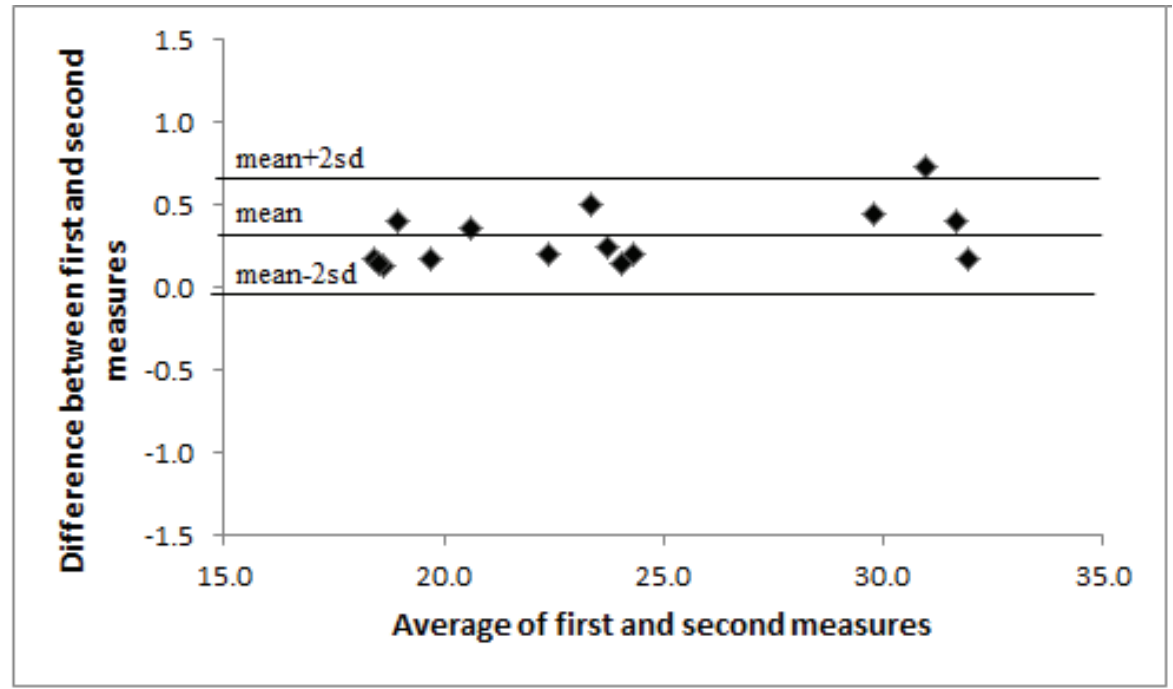

b.

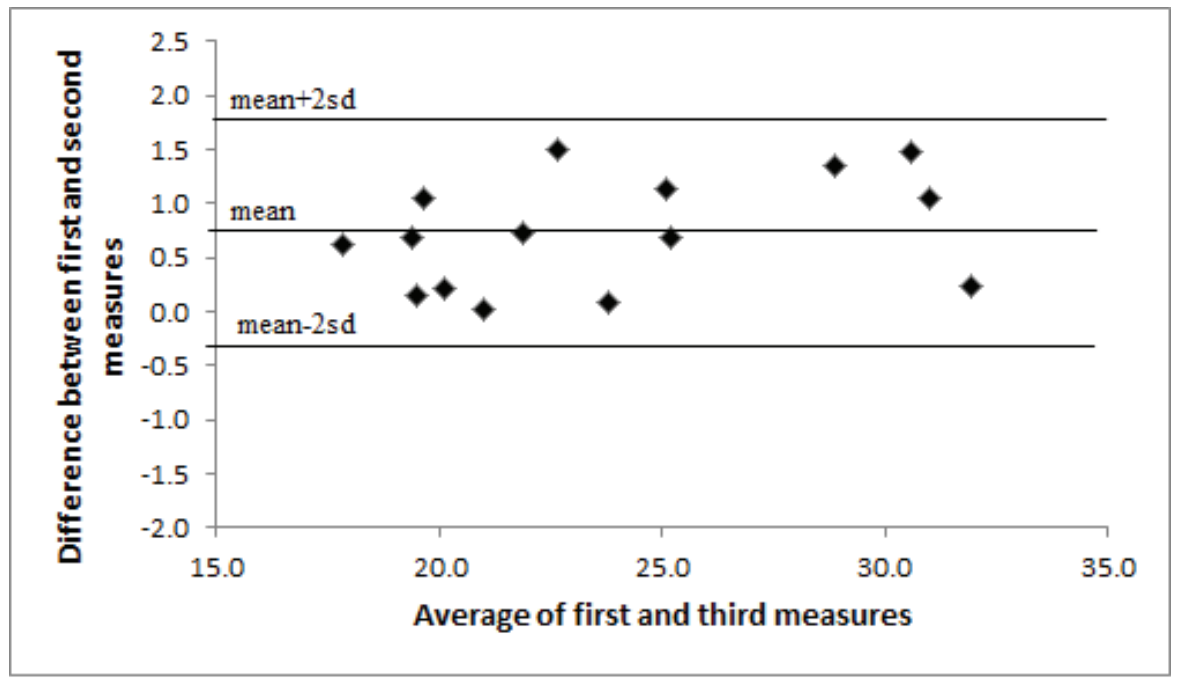

Fig. 2. Differences between pairs of measures plotted against the mean of those pairs of measures for RMS activity of muscles (a. measured on the same day; b. measured over 1 week)

0.29 with $95 \%$ CI of $-0.05-0.64$ and 0.73 with $95 \%$ CI of $-0.29-1.76$ for within-day and between-day measurements, respectively, which indicate a good level of agreement between the two measurements. Figure 3 demonstrates a comparison of time broadness between the first and second measurements (within-day) and first and third measurements (between-day). The mean differences were 0.15 with $95 \%$ CI of $-0.26-0.56$ and 0.39 with $95 \%$ CI of $-0.25-1.05$ for within-day and between-day measurements, respectively, which indicate a good level of agreement between the two measurements [30].

\section{Discussion}

This study examined the reliability of the EMG assessment procedure for ten selected shoulder muscles during the overhead volleyball throw. The results of this study indicated that the test protocol used in this research could reliably measure the RMS and time broadness of EMG activity of shoulder muscles during the overhead volleyball throw.

Although the overhead throw is known and recommended as a valid functional test in volleyball [24], there is a lack of evidence regarding the reproducibility of EMG recordings during this task so far. Researchers previously investigated the EMG activity of the thrower's shoulder muscles $[2,10,16,22,23]$, and also specifically during the spike and overhead serve $[14,15,17,18,26]$. In Kelly and colleague's EMG study (2002) of shoulder muscles during the functional football throw in professional players little variation in electromyographic activation between subjects during the phases of throw motion was observed, but they provided no information about the reliability of EMG. However, the pattern of RMS activity in our study is highly consistent with RMS activity of their study, which represented higher activity of the pectoralis major 
a.

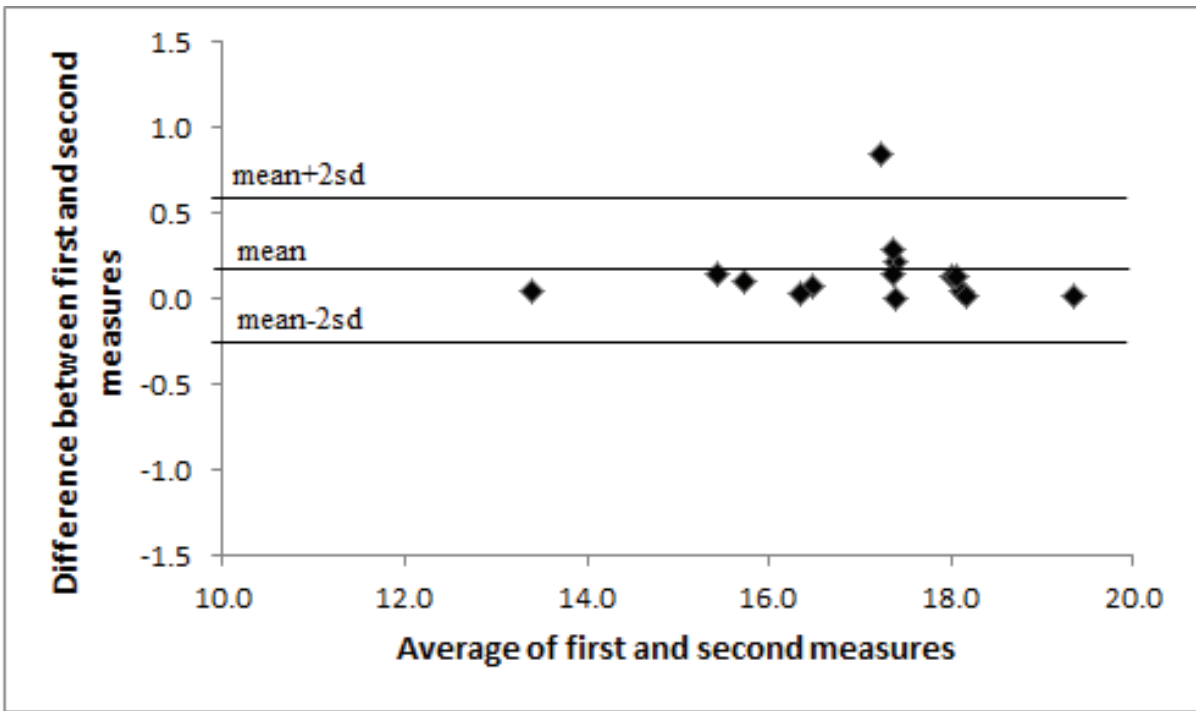

b.

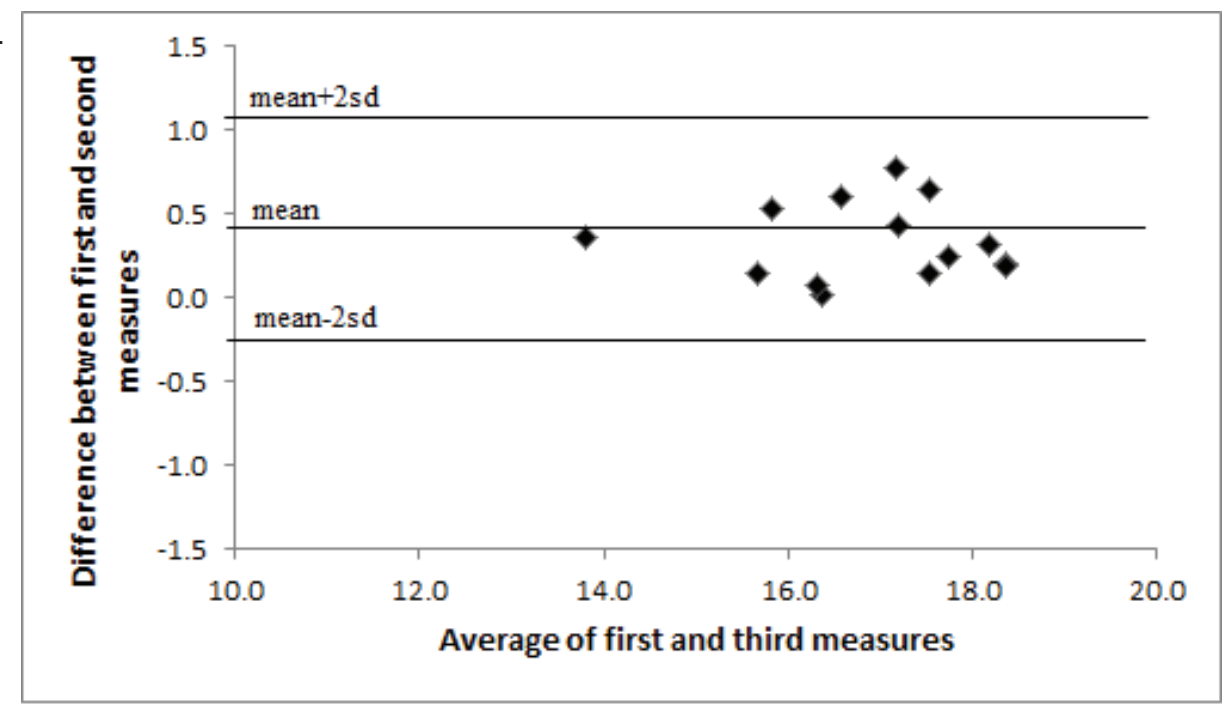

Fig. 3. Differences between pairs of measures plotted against the mean of those pairs of measures for time broadness activity of muscles (a. measured on the same day; b. measured over 1 week)

and lower activity of the posterior deltoid in the acceleration phase. It is also noteworthy that the lower trapezius had the lowest activity among other muscles in our study, which was not recorded in the Kelly et al. study. Illyés and Kiss in 2009 and 2007 reported the RMS activity levels and time broadness of shoulder muscle activity in professional javelin throwers during the overhead throw of a tennis ball $[14,15]$, and they found dysfunctional neuromuscular control of the rotator cuff muscles in patients with shoulder instability [14] and lower time broadness of muscle activity in healthy shoulders compared to shoulders with instability $[14,15]$. Nevertheless, the RMS activity of the muscles did not have quite the same pattern as ours in their study. Regarding the different technique of throwing a tennis ball versus spiking volleyball (more powerful humeral rotation and less powerful flexion and horizontal adduction in throwing a tennis ball), they found higher activity of the posterior deltoid muscle and lower activity of the anterior deltoid muscle. Although retesting over days and relocating EMG electrodes seemed to be sources of error, they observed lower time broadness of muscle activity and higher RMS activity of symptomatic shoulders after therapeutic exercises [14].

Reliability of measuring normalized RMS activity of shoulder muscles has been investigated in a few studies $[1,21,29]$. However, the measurement methods, selected population (athletes, non-athletes), selected test position (static or dynamic), selected muscle (SS, IS, UT, LT, PM, $\mathrm{SA}, \mathrm{AD}, \mathrm{PD}, \mathrm{BB}, \mathrm{TB})$ and statistical approaches have been quite varied across these studies. Andersen and colleagues (2014) demonstrated the between-day reliability of measuring upper trapezius and anterior deltoid RMS activity in static positions (isometric submaximal contractions during flexion $45^{\circ} / 90^{\circ}$, abduction $45^{\circ} / 115^{\circ}$, and 
internal/external rotation) and obtained acceptable reliability (ICC $=0.82-0.92)$ and low limits of agreement with near to zero mean difference of the measurements (mean difference $=0.25-0.70$ ). By contrast, in this study, lower ICC values $(0.79$ and 0.80$)$ and higher mean difference of the between-day measurements (1.67-1.73) were observed for both muscles. In two other studies, the reliability was examined over a dynamic position [21, 29]. Ludewig and Cook (2000) assessed average normalized RMS values and reported moderate to high $(\mathrm{ICC}=0.73-$ $0.90)$ reliability of measuring RMS activity of upper trapezius, lower trapezius and serratus anterior muscles in scaption movement after 2 days, and they concluded that scaption movement with the speed of one repetition per 4 seconds can be reliably measured and used as an outcome measure in the rehabilitation of patients with symptoms of shoulder impingement. They observed higher ICC (0.82-0.90) of EMG activity of lower trapezius compared with upper trapezius ( $\mathrm{ICC}=0.78-0.88$ ) and serratus anterior $(0.73-0.89)$ muscles. In the current study higher ICC was found in UT $($ ICC $=0.79)$ compared to LT $(\mathrm{ICC}=0.71)$ and SA $(\mathrm{ICC}=0.70)$. In comparison to these two studies, the higher demands of a dynamic rapid movement and longer between-day duration (7 days) of the present study could be responsible for the different ICC values.

On the other hand, lower ICC values do not represent lower agreement of the measurements. The absolute agreement which is defined by the SEM, limits of agreement and mean difference of each pair of measurements are more significant factors. As demonstrated in Table 3, in this study, small SEM values of RMS activity and time broadness measures were obtained both for within-day and between-day measurements. The mean differences and limits of agreement also showed low values, indicating high agreement of the measurements. Among previous studies, only one reliability study was found investigating these agreement factors in shoulder EMG. Seitz and Uhl (2012) investigated the within-day and between-day reliability of RMS activity of anterior deltoid, upper trapezius, lower trapezius and serratus anterior muscles in a slow scaption movement (one repetition per 4 seconds). They found the test-testing procedure to be reliable and obtained higher within-day reliability $(\mathrm{ICC}=0.96-0.99 ; \mathrm{SEM}=1.0-2.0$; $\mathrm{MDC}=1.3-2.9)$ compared to between-day reliability $(\mathrm{ICC}=0.59-0.86 ; \mathrm{SEM}=2.3-8.3 ; \mathrm{MDC}=3.2-11.7)$, but regarding the results of the between-day measurements (low ICC values and high SEM and MDC values), the reliability is questionable. In their study, despite the movements being performed slowly in a controlled position and far slower than the high risk rapid arm movement in a volleyball spike or serve, the between-day reliability results were lower than those found in the present study, which may be attributed to more precise electrode placement and skin preparation in this study.

Overall, the results of this study suggest that the activity of shoulder muscles can be reliably assessed (over within-day and/or between-day sessions) during the overhead volleyball throw with the described procedure both in amplitude domain (normalized RMS average) and time domain (time broadness of the activities). However, further studies are recommended to support the findings of the present study.

\section{Conflict of interest: Authors state no conflict of interest.}

\section{References}

1. Andersen K.S., Christensen B.H., Samani A., Madeleine P. (2014) Between-day reliability of a hand-held dynamometer and surface electromyography recordings during isometric submaximal contractions in different shoulder positions. J. Electromyogr. Kinesiol., 24(5): 579-587. DOI: 10.1016/j.jelekin.2014.05.007.

2. Barden J.M., Balyk R., Raso V., Moreau M., Bagnall K. (2005) Atypical shoulder muscle activation in multidirectional instability. Clin. neurophysiol., 116(8): 1846-1857. DOI: 10.1016/j.clinph.2005.04.019.

3. Bland J.M. Altman D.G. (2010) Statistical methods for assessing agreement between two methods of clinical measurement. Int. J. Nurs. Stud., 47(8): 931-936. DOI: 10.1016/j.ijnurstu.2009.10.001.

4. Burkhart S.S., Morgan C.D., Kibler W.B. (2003) The disabled throwing shoulder: spectrum of pathology Part I: pathoanatomy and biomechanics. Arthroscopy, 19(4): 404-420.

5. Burkhart S.S., Morgan C.D., Kibler W.B. (2003) The disabled throwing shoulder: spectrum of pathology part II: evaluation and treatment of SLAP lesions in throwers. Arthroscopy, 19(5): 531-539. DOI: 10.1053/ jars.2003.50139.

6. Burkhart S.S., Morgan C.D., Kibler W.B. (2003) The disabled throwing shoulder: spectrum of pathology Part III: The SICK scapula, scapular dyskinesis, the kinetic chain, and rehabilitation. Arthroscopy, 19(6): 641-661. DOI: 10.1016/S0749-8063(03)00389-X.

7. Cohen J. (1988) Statistical power analysis for the behavioral sciences. Lawrence Erlbaum Associates, Inc, New Jersey.

8. Criswell E. (2010) Cram's introduction to surface electromyography. Jones \& Bartlett Publishers, Sudbury, MA.

9. Hallal C.Z., Marques N.R., Gonçalves M. (2011) Electromyographic ratio of shoulder stabilizer muscles during performance of exercises with oscillatory poles. Rev. 
Bras. Med. Esporte, 17: 31-35. DOI: 10.1590/S151786922011000100006.

10. Henry T.J. (1997) An electromyographic analysis of dynamic stabilizing exercises for the shoulder, University of Pittsburgh, Ann Arbor, pp:71-71.

11. Hess S.A., Richardson C., Darnell R., Friis P., Lisle D., Myers P. (2005) Timing of rotator cuff activation during shoulder external rotation in throwers with and without symptoms of pain. J. Orthop. Sports Phys. Ther, 35(12): 812-820. DOI: 10.2519/jospt.2005.35.12.812.

12. Hislop H., Avers D., Brown M. (2014) Daniels and Worthingham's Muscle Testing: Techniques of Manual Examination and Performance Testing. Saunders, Philadelphia, WB.

13. Huxel K.C. (2005) Gender differences in muscle recruitment and stiffness regulation strategies of the shoulder, Temple University, Ann Arbor, pp: 216-216.

14. Illyés Á., Kiss J., Kiss R.M. (2009) Electromyographic analysis during pull, forward punch, elevation and overhead throw after conservative treatment or capsular shift at patient with multidirectional shoulder joint instability. J. Electromyogr. Kinesiol., 19(6): e438-e447. DOI: 10.1016/j.jelekin.2008.09.008

15. Illyés Á. Kiss R.M. (2007) Electromyographic analysis in patients with multidirectional shoulder instability during pull, forward punch, elevation and overhead throw. Knee Surg. Sports Traumatol. Arthrosc., 15(5): 624-631. DOI: 10.1007/s00167-006-0163-1.

16. Jaggi A., Noorani A., Malone A., Cowan J., Lambert S., Bayley I. (2012) Muscle activation patterns in patients with recurrent shoulder instability. Int. J. Shoulder Surg., 6(4): 101. DOI: 10.4103/0973-6042.106221.

17. Kelly B.T., Backus S.I., Warren R.F., Williams R.J. (2002) Electromyographic analysis and phase definition of the overhead football throw. Am. J. Sports Med., 30(6): 837-844. DOI: 10.1177/03635465020300061401.

18. Kibler W.B., Chandler T.J., Shapiro R., Conuel M. (2007) Muscle activation in coupled scapulohumeral motions in the high performance tennis serve. Br. J. Sports Med., 41(11): 745-749. DOI: 10.1136/bjsm.2007.037333.

19. Kibler W.B., Kuhn J.E., Wilk K., Sciascia A., Moore S., Laudner K., Ellenbecker T., Thigpen C., Uhl T. (2013) The disabled throwing shoulder: Spectrum of pathology - 10-year update. Arthroscopy, 29(1): 141-161. e126. DOI: $10.1016 /$ j.arthro.2012.10.009.

20. Kiss J., Damrel D., Mackie A., Neumann L., Wallace W. (2001) Non-operative treatment of multidirectional shoulder instability. Int. Orthop., 24(6): 354-357. DOI: $10.1007 / \mathrm{s} 002640000202$.
21. Ludewig P.M. Cook T.M. (2000) Alterations in Shoulder Kinematics and Associated Muscle Activity in People With Symptoms of Shoulder Impingement. Phys. Ther, 80(3): 276-291. DOI: 10.1093/ptj/80.3.276.

22. Morris A.D., Kemp G.J., Frostick S.P. (2004) Shoulder electromyography in multidirectional instability. J. Shoulder Elbow. Surg., 13(1): 24-29. DOI: 10.1016/j. jse.2003.09.005

23. Myers J.B., Ju Y.-Y., Hwang J.-H., Mcmahon P.J., Rodosky M.W., Lephart S.M. (2004) Reflexive muscle activation alterations in shoulders with anterior glenohumeral instability. Am. J. Sports Med., 32(4): 1013-1021. DOI: 10.1177/0363546503262190.

24. Palao J.M. Valadés D. (2012) Validity of the standing spike test as a monitoring protocol for female volleyball players. Biol. Sport, 29(4): 281-284. DOI: 10.5604/20831862.1019666.

25. Richman J., Makrides L., Prince B. (1980) Research methodology and applied statistics. Part 3: measurement procedures in research. Physiother. Can., 32(4): 253-257.

26. Rokito A.S., Jobe F.W., Pink M.M., Perry J., Brault J. (1998) Electromyographic analysis of shoulder function during the volleyball serve and spike. J. Shoulder Elbow. Surg., 7(3): 256-263. DOI: 10.1016/S1058-2746(98)90054-4.

27. Santos M.J., Belangero W.D., Almeida G.L. (2007) The effect of joint instability on latency and recruitment order of the shoulder muscles. J. Electromyogr. Kinesiol., 17(2): 167-175. DOI: 10.1016/j.jelekin.2006.01.010.

28. Sciascia A., Kuschinsky N., Nitz A.J., Mair S.D., Uhl T.L. (2012) Electromyographical comparison of four common shoulder exercises in unstable and stable shoulders. Rehabil. Res. Pract. DOI: 10.1155/2012/783824.

29. Seitz A.L. Uhl T.L. (2012) Reliability and minimal detectable change in scapulothoracic neuromuscular activity. J. Electromyogr. Kinesiol., 22(6): 968-974. DOI: 10.1016/j.jelekin.2012.05.003.

30. Weir J.P. (2005) Quantifying test-retest reliability using the intraclass correlation coefficient and the SEM. J. Strength Cond. Res., 19(1): 231-240. https://journals. lww.com/nsca-jscr/Abstract/2005/02000/QUANTIFYING_TEST_RETEST_RELIABILITY_USING_ THE.38.aspx

\section{Received 02.06.2018 \\ Accepted 15.10.2018}

(C) University of Physical Education, Warsaw, Poland 